\title{
Mechanical Complications of Acute Myocardial Infraction
}

\author{
Wei Wang and Anson Cheung
}

\subsection{Introduction}

With timely reperfusion, myocardial loss following myocardial infarction (MI) can be significantly reduced and may limit the incidence of mechanical complications. However, with improving treatment of those with larger or delayed presentation MI, appropriate management of mechanical complications remains a key consideration for those working in heart attack centers.

\subsection{Ischemic Ventricular Septal Defect}

\subsubsection{Incidence and Pathophysiology}

The incidence of post-infarction ventricular septal defect (VSD) is reported as $1-2 \%$ after acute myocardial infarction (MI). However, the true incidence may be decreasing in modern era with the advancement of early interventions after acute myocardial infarction including thrombolytic treatment, early revascularization with percutaneous coronary angioplasty, or stenting and coronary bypass grafting. Nonetheless, timely and appropriate management of this important complication of MI can be lifesaving.

The timing to develop post-infarction VSD is variable, in a few hours and up to 2 weeks with an average time of 2-4 days after acute MI. It is usually associated with complete occlusion of a single coronary artery with poor collateral vessels. About 2/3 of post-infarction VSDs are located in the anteroapical septum, while the

W. Wang $\cdot$ A. Cheung $(\triangle)$

University of British Columbia, Vancouver, BC, Canada

e-mail:wwang7@ providencehealth.bc.ca; acheung@providencehealth.bc.ca 
remaining in the posterior septum, from acute left anterior artery (LAD) and dominant right coronary artery (RCA) or circumflex artery (LCx) occlusions, respectively.

The natural history of untreated VSD is extremely poor, carrying a mortality rate of $25 \%$ within $24 \mathrm{~h}$ and $80 \%$ within 4 weeks. The early development of congestive heart failure (CHF) leading to cardiogenic shock is the primary cause of death. Ventricular dysfunction can be primarily left or right, depending on the territory infracted and the location of the septal rupture. Especially in posterior infarct, significant mitral valve regurgitation (MR) can play an important role in the development of heart failure, resulting in elevated left ventricular end-diastolic pressure (LVEDP) and pulmonary edema. The degree of left-to-right shunt depends on the size of the VSD and pressure gradient across the septum. If the shunt is large, the normally compliant right ventricle (RV) will not tolerate the sudden increase in load, and the development of significant RV failure pursues. As a result, severe biventricular failure is not uncommon in patients with post-infarction VSD.

\subsubsection{Presentation and Diagnosis}

Typical presentation of post-infarction VSD includes the presence of a new holosystolic murmur radiating to the axilla a few days post MI. It is often accompanied by an abrupt hemodynamic deterioration, i.e., signs of $\mathrm{CHF}$ and cardiogenic shock.

Electrocardiography (ECG) may show ST elevation and/or Q wave in accordance with location of transmural MI. Chest radiography (CXR) often shows acute pulmonary edema. Currently, transthoracic echocardiography (TTE) with color Doppler is the quickest and most definitively diagnostic method for post-infarction VSD. It can delineate the size, location, presence, and degree of shunt. In addition, TTE can identify and quantify the degree of MR and rule out other causes of MR, such as secondary to papillary muscle rupture and primary MR. Other valuable information include the presence of other valvular pathology, the degree of ventricular dysfunctions, free wall rupture, cardiac infront of tamponade, pulmonary embolism, and aortic dissection/rupture. As many patients now undergo rapid left heart catheterization to permit primary percutaneous coronary intervention (PCI) even increasingly among late presenters - the finding of a compatible murmur during clinical evaluation may prompt left ventriculography which may prove diagnostic for VSD. The role of primary PCI or concomitant coronary artery bypass grafting in the setting of VSD is debatable as myocardial damage is already transmural and residual viability therefore questionable.

Right heart catheterization is usually not required, but a step-up in the oxygen saturation between the right atrium (RA) and pulmonary artery (PA) indicates leftto-right shunt. The right heart catheterization can also calculate the pulmonary to systemic flow ratio (Qp/Qs). 


\subsubsection{Timing of Surgery}

The natural history of untreated post-infarction VSD is very poor with a $25 \%$ mortality within 24 h, $50 \%$ mortality within 1 week, $80 \%$ within 1 month, and $97 \%$ at 1 year [1]. The presence of post-infarction VSD is therefore an indication for urgent interventions. Intervention includes definitive surgical correction, mechanical circulatory support (MCS), and device closure of VSD. Cardiogenic shock in the setting of postinfarction VSD is a surgical emergency. In patients with cardiogenic shock, early MCS using extracorporeal membrane oxygenation (ECMO) or other short-term devices should be initiated to stabilize hemodymics and correct metabolic derangements. Furthermore, it can bridge the patient to decision regarding the best therapeutic option. Percutaneous device closure rarely works in the acute setting as the VSD is frequently serpentines, the infarcted and peri-infarct myocardium is necrotic and fragile. Given friable myocardium at the rim of the VSD, the use of percutaneous closure device can result in significant residual shunt and device embolization.

\subsubsection{Preoperative Care}

Medical management is aimed to improve cardiac output and reduce the left-toright shunt by reducing systemic vascular resistance and LV pressure. Inotropes and diuretics are often used. Placement of an intra-aortic balloon pump (IABP) and other short-term MCS device can be helpful.

\subsubsection{Surgical Techniques}

Surgical principles include elimination of left-to-right shunt, preservation of LV function, and revascularization when possible. Small anterior and apical defects can be closed by suturing the free wall of the RV and LV, sandwiching the septum. For larger defects, generally the two commonly employed surgical techniques are infarctectomy and infarct exclusion. Infarctectomy technique involves removal of left ventricular infarction area and closure of VSD without tension. Infarct exclusion technique, on the other hand, does not remove any infarct tissue. Principles of both techniques include good myocardial protection during the operation, suture placement through non-infarcted tissue to maximize suture line integrity, and minimizing tension with the use of large prosthetic patches. Infarct exclusion techniques are more popular and thought to have the advantages of preserving left ventricular geometry and function. Meticulous medical management intraoperatively is critical, and intraoperative transesophageal echo (TEE) is mandatory. After the establishment of cardio-pulmonary bypass (CPB) and arresting the heart, the heart is inspected and the infarcted area exposed. In primary infarctectomy repair, the infarcted wall is incised (Fig. 20.1). After the resection of necrotic septal myocardium, pledgeted interrupted horizontal mattress sutures are placed around the 
defect. Subsequently, sutures are then passed through the septum from right to left through a felt strip. Closure of the ventriculotomy is reinforced with felt strips. Infarctectomy may also be performed with bovine pericardial patch repair (Fig. 20.2). As previously described, after the resection of infarcted tissue, pledgeted interrupted horizontal mattress sutures are placed around the defect. Sutures are then passed through a large bovine pericardial patch. All sutures are pledgeted once again and then tied down. The edges of the ventriculotomy are reapproximated with a double-layer buttressed closure with Teflon felt. Biological glue is often used to ensure complete hemostasis.
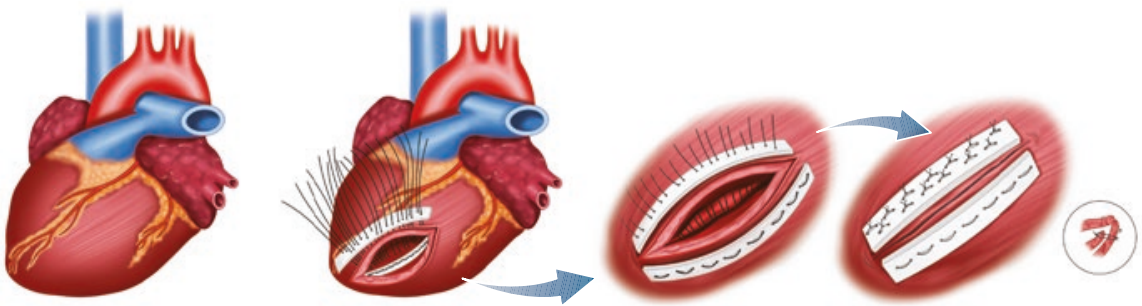

Fig. 20.1 Infarctectomy technique for anterior VSD repair with primary closure. [Courtesy of Miss Christina YS Wong]

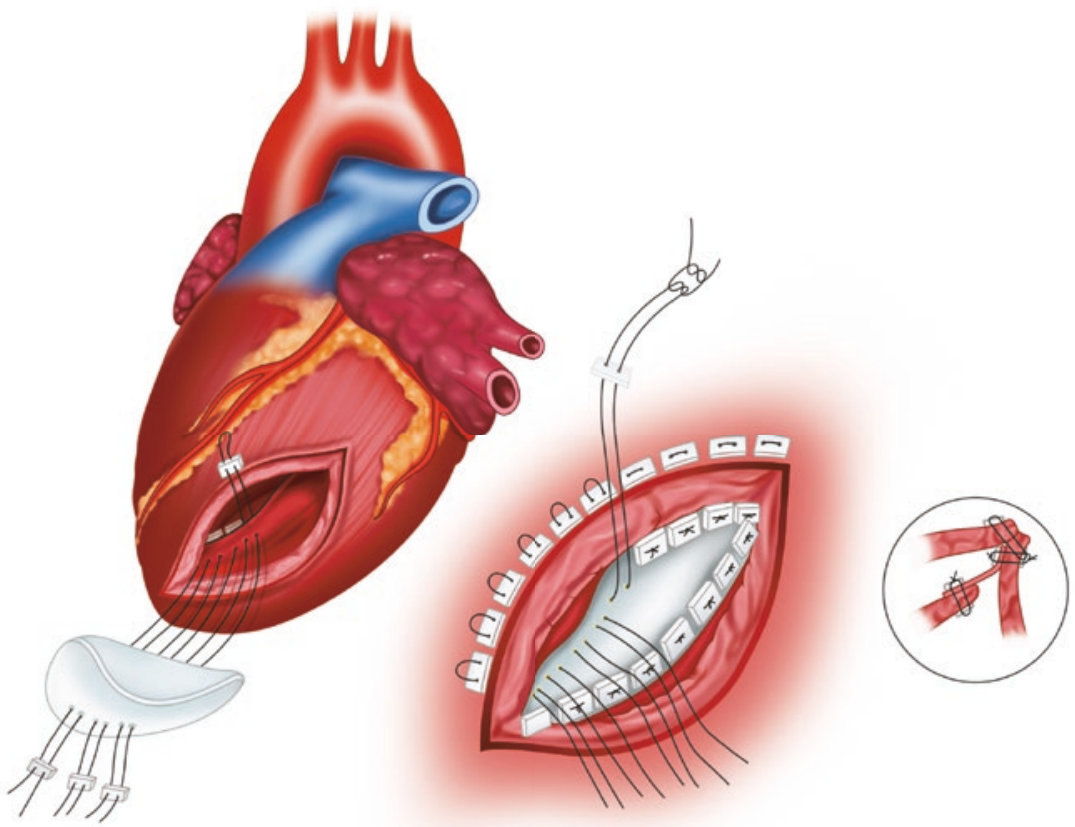

Fig. 20.2 Infarctectomy with bovine pericardial patch. [Courtesy of Miss Christina YS Wong] 
Infarct exclusion techniques differ from infarctectomy where no infarcted tissue is being removed (Fig. 20.3). A trans-infarct incision is made and the extent of the infarct inspected. A large bovine pericardial patch covering is used to exclude defect from LV cavity; placements of suture onto healthy septal tissue are crucial in the integrity of the repair. Closure of the ventriculotomy is then closed as previously described. Repair of posterior ischemic VSD can sometimes be technically more challenging than anterior as exposure is less ideal. The infarct exclusion technique can provide a more tension-free repair and is believed to be better in preserving LV and RV geometry and function.

A third surgical technique, the right atrium approach, has been described for the post-infarction VSD (Fig. 20.4). It is used more commonly in posterior VSD repair. It has the benefit of not performing a left ventriculotomy. Repair is done through the tricuspid valve as performed in congenital ventricular septal defect patients [2]. However, this technique has not gained popularity for a number of reasons. Firstly,
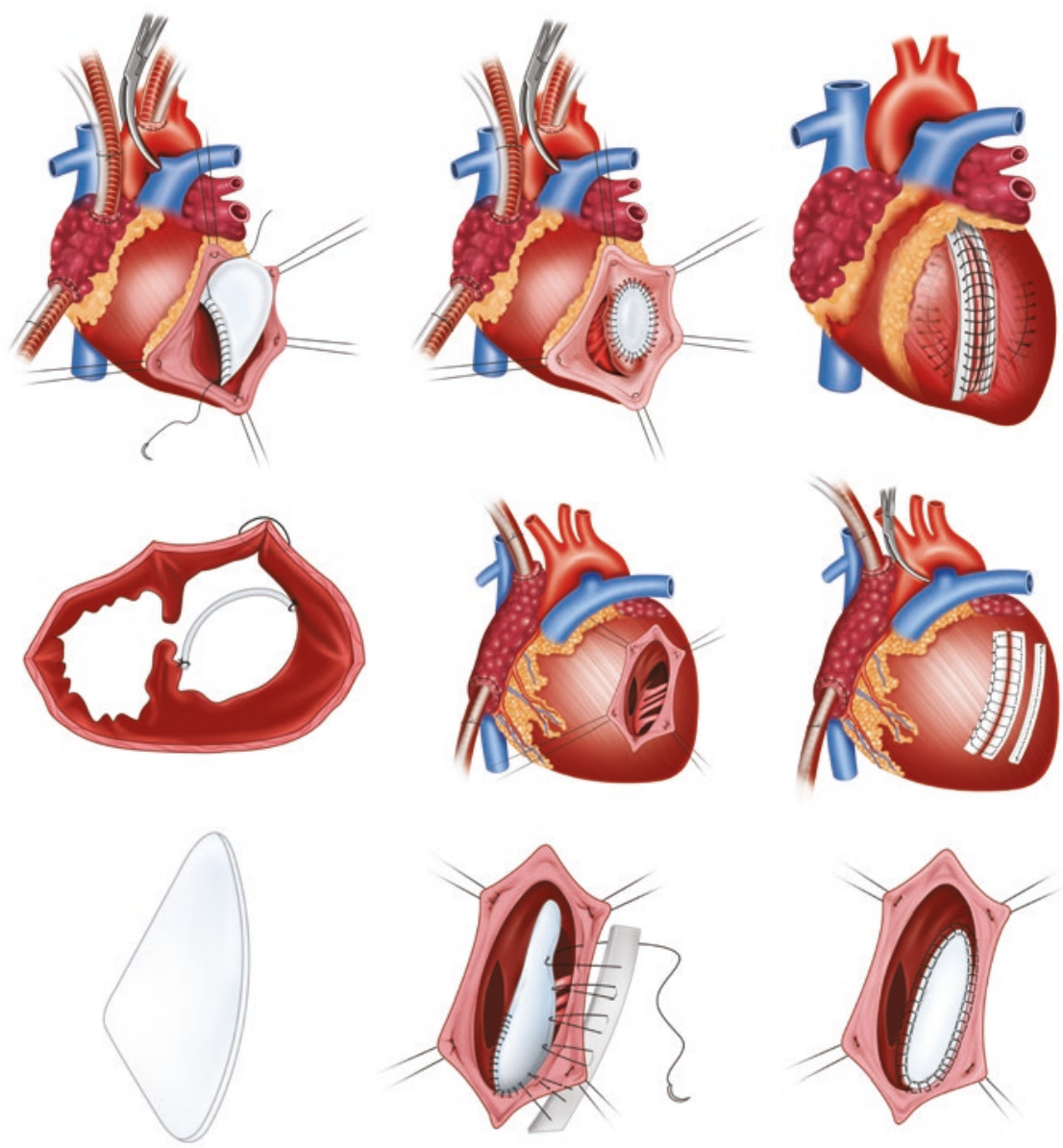

Fig. 20.3 Infarct exclusion for anterior (left) and posterior (right) post-infarction VSD. [Courtesy of Miss Christina YS Wong] 
Fig. 20.4 Right atrium approach for posterior VSD repair. [Courtesy of Miss Christina YS Wong]
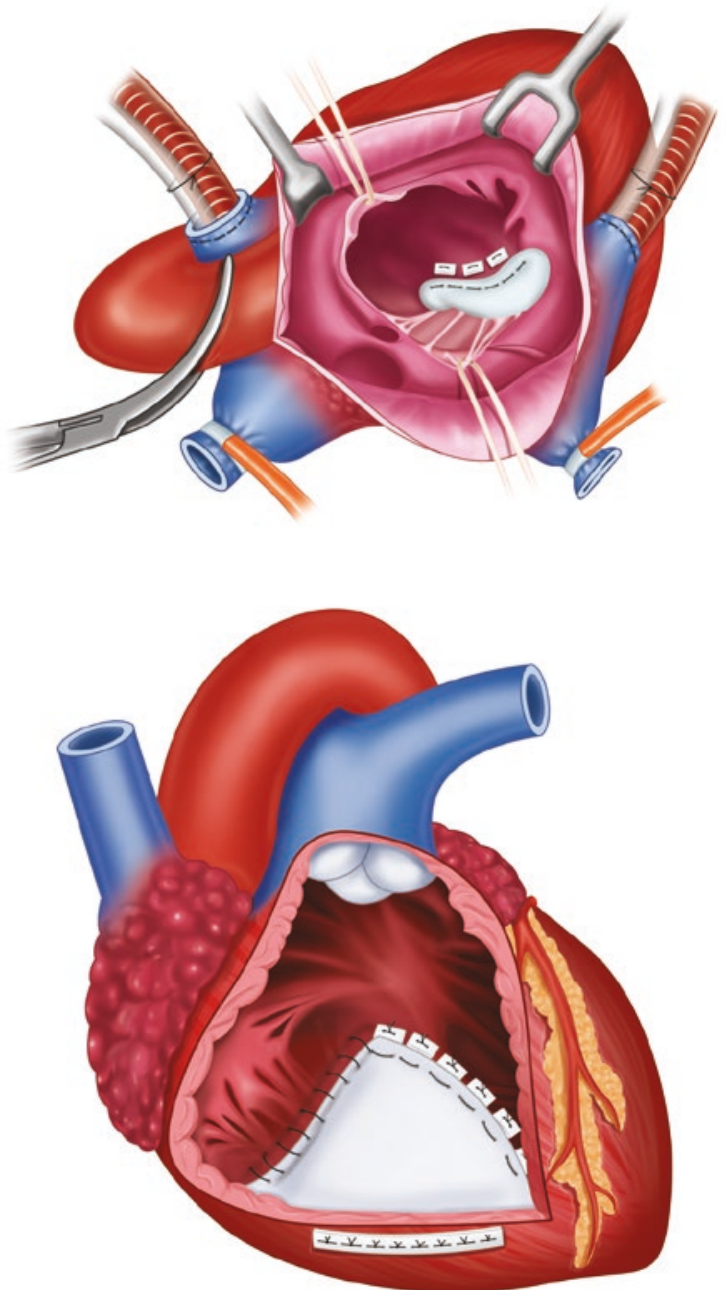

the exposure of VSD through the right atrium is often suboptimal. Secondly, placement of repair sutures can be difficult requiring detachment of the septal leaflet of tricuspid valve and the need for tricuspid valvuloplasty. Thirdly, putting a large patch from the right side of the ventricular septum can be difficult, especially in acute phase. This technique is best performed in patients 2-4 weeks after presentation of VSD, where a fibrotic rim will often develop.

\subsubsection{Transcatheter Device Closure}

Transcatheter device is widely used in patients with congenital VSD. However, the role of transcatheter device in post-infarction VSD is unclear. Limited data is available and most of the case studies had small number of patients. When device closure is 


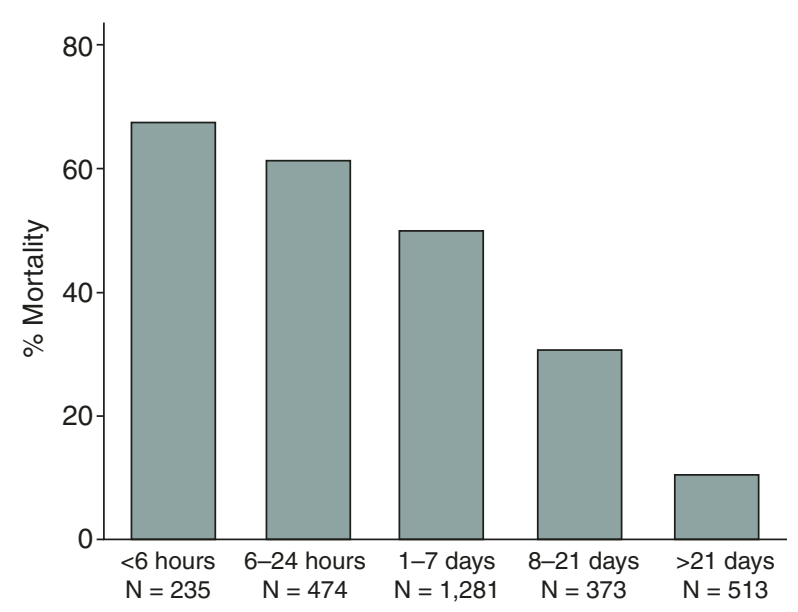

Fig. 20.5 Operative mortality rate stratified by timing of VSD repair [5]

performed in acute phase of post-infarction VSD, the 30-day mortality can be as high as $65 \%$ [3]. A study from Mayo Clinic also showed the predictors of adverse events from transcatheter device closure of post-infarction VSD included cardiogenic shock and acute phase post MI. At present, device closure is limited in selected patients 4-6 weeks after post-infarction VSD, with the fibrotic rim is well developed.

\subsubsection{Mechanical Circulatory Support Involvement (ECMO, LVAD)}

With the advancement of mechanical circulatory support, patients with severe endorgan damage deemed too sick to undergo operative repair may be candidates for a delayed repair. Interval treatment which may involve placement of short-term MCS devices will help improve end-organ dysfunction and allow for maturation of the infarcted tissue. Durable MCS devices can be used, however, not encouraged, due to complexity of the implant procedure, the high mortality associated with postinfarct VSD, and the cost of the device (Fig. 20.5).

\subsubsection{Postoperative Management and Surgical Results}

Postoperative care centers on ensuring adequate cardiac output for end-organ perfusion and correcting metabolic derangements. Pharmacologic agents such as epinephrine and milrinone may help to augment contractility. After separating from $\mathrm{CPB}$, right-sided heart failure is common; inhaled nitric oxide, prostaglandin, and milrinone can be helpful in this setting. Some patients may require temporary right ventricular assist device postoperatively. The utility and effectiveness of IABP in this setting are not well established, and its use in cardiogenic shock is not recommended [4]. 


\subsubsection{Outcome}

Operative mortality is relatively high which ranges from 30 to $50 \%$. Risk factors for early mortality after surgical intervention include persistent low cardiac output state and recurrent or residual VSD. While a residual defect with a significant shunt in an unstable patient should be imminently addressed, delayed repair is favored in a hemodynamically stable patient with a residual VSD.

Arnaoutakis et al. analyzed and reported the outcome of 2876 patients with postinfarction VSD who underwent surgical repair from the Society of Thoracic Surgery database. The operative mortality was $54.1 \%$ when repair occurred within 7 days from myocardial infarction, and only $18.4 \%$ when repair was performed more than 7 days from infarction [5]. These results may suggest mechanical circulatory support could play an important role in delaying surgical repair to achieve much better outcome in hemodynamically unstable patients. Alternatively, this finding can be explained by selection bias.

\subsection{Acute Mitral Valve Regurgitation Secondary to Papillary Muscle Rupture}

\subsubsection{Pathophysiology}

Acute mitral valve regurgitation secondary to papillary muscle rupture is a catastrophic complication after acute MI. Approximately $1-5 \%$ of death after acute MI is due to acute mitral valve regurgitation as a result of papillary muscle rupture. As in post-infarction VSD, the incidence of acute papillary muscle rupture is decreasing with early reperfusion interventions. Typically, the anterolateral papillary muscle has dual blood supply by the LAD and LCx, whereas the posteromedial papillary muscle has single blood supply by either the dominant RCA or the circumflex artery in a left dominant circulation. Hence the posteromedial papillary muscle is more susceptible to ischemia and consequent rupture accounting for $2 / 3$ of all cases and are more commonly associated with an acute inferior STEMI.

\subsubsection{Presentation and Diagnosis}

Post-infarction acute MR usually occurs 2-7 days after MI. It carries a very high mortality of 50-75\% without surgical intervention and 20-25\% with surgical intervention. A new holosystolic murmur in conjunction with cardiovascular collapse several days after MI should raise the suspicion of papillary muscle rupture. The major differential is post-infarction VSD. Unlike an acute VSD, MR is best heard at the apex rather than the left sternal border and does not have an associated thrill. Papillary muscle rupture results in mitral valve flail leaflet and severe mitral valve regurgitation. In a normal left atrium (LA) with a relatively low compliance, acute 
mitral regurgitation results in sudden elevation of LA pressure leading to rapid development of pulmonary edema and cardiogenic shock.

EKG may show signs of an inferior MI, and chest radiography may demonstrate severe pulmonary edema. TTE with color Doppler is the golden standard for diagnosis. It can confirm mitral valve prolapse with flailing chordae or papillary muscle, quantify the degree of MR and ventricular function, and rule out postinfarction VSD. Development of multi-organ failure is common in patients with acute MR.

\subsubsection{Preoperative Considerations}

Acute MR often results in rapid hemodynamic collapse. Intubation and mechanical ventilation may be required for respiratory failure. Inotropes and diuretics can stabilize some patients; however, in unstable patients with evidence of cardiogenic shock or severe end-organ failure, early MCS with short-term devices can be lifesaving.

\subsubsection{Operative Techniques}

Emergent mitral valve replacement (MVR) is most commonly undertaken with concomitant coronary revascularization. Even in patients who received satisfactory percutaneous coronary interventions, isolated MVR should be performed emergently in papillary muscle rupture cases. Successful MV repair cases have been reported with reimplantation of papillary muscle, chordal transfer, and artificial chord implantation; however, repair is not recommended as the early failure rate is very high in the acute setting.

Surgical technique emphasizes on a swift operation with good myocardial protection, and MVR is generally performed via a median sternotomy. Arterial and vein graft conduits are harvested if concomitant CABG is needed. Routine cardiopulmonary bypass cannulation and antegrade and retrograde cardioplegia are routinely used. For patients with concomitant $\mathrm{CABG}$, distal coronary anastomoses are performed first. Mitral valve exposure can be difficult in acute setting as the LA is often small and not dilated. Both mechanical and tissue valve may be used. It is important to preserve the chordae and leaflets (especially posterior leaflet) as much as possible to better preserve the left ventricular geometry.

\subsubsection{Results}

The surgical mortality of MVR in patients with acute MR secondary to papillary muscle rupture can be as high as $30 \%$. Literature-supported concomitant CABG with MVR has an improved long-term survival compared to MVR alone [6]. 


\subsection{Left Ventricular Free Wall Rupture}

\subsubsection{Pathophysiology}

Free wall ventricular rupture occurs in $2-4 \%$ of patients presenting with an acute MI. Autopsy data showed the incidence could be as high as $11 \%$ [1]. Like ventricular septal defect, patients with LV free wall rupture commonly have transmural ST elevation MI. Rupture tends to occur at the junction of viable and necrotic myocardium when the infarction expands. Early thrombolytic therapy is associated with decreased incidence of free wall rupture after acute MI.

LV free wall rupture can be classified as acute, subacute, and chronic. Acute LV free wall rupture may cause severe hemodynamic compromise secondary to cardiac tamponade, often leading to sudden death. Subacute rupture indicates a small tear on LV free wall that may be temporarily clotted, contained, and not leading to immediate death. Chronic LV free wall ruptures commonly occur in the posterior wall. It is associated with very small leakage of blood that is walled-off by pericardium and the subsequent formation of a false aneurysm. Surgical interventions are indicated in subacute and chronic LV free wall ruptures.

\subsubsection{Presentation and Diagnosis}

Cardiac tamponade and cardiogenic shock are the most common presentations in patients with LV free wall rupture. Patients can have signs of tamponade including increased in jugular venous pressure and pulsus paradoxus. An urgent echocardiography is the choice for diagnosis in unstable patient. In stable and chronic patients, cardiac CT with contrast can provide better anatomical information and may aid surgical planning.

\subsubsection{Surgical Techniques for Repair}

Subacute LV free wall rupture requires emergency surgical repair. Pericardiocentesis might be helpful to stabilize patient's hemodynamic status while preparing an operating room. An IABP or other short-term MCS device is commonly necessary for preoperative stabilization. A variety of repair techniques have been described. Rupture closure with horizontal mattress sutures buttressed with Teflon felts is widely used. An additional bovine pericardial, Dacron, or PTFE patch can also be used to reinforce the suture line. Simple placement of a large pericardial patch on the ruptured surface with Bio-glue can be an option in cases where the myocardial tissue is too fragile to be repaired safely.

Chronic LV free wall rupture with false aneurysm does not require emergency surgery. Cardiac CT and MRI can better delineate the anatomical defect. The 
aneurysm neck can be identified readily and contains fibrotic scar tissue; primary closure can be performed on an elective basis. Reconstruction with Dacron or bovine pericardial patch can also be used.

\subsubsection{Results}

LV free wall rupture is usually lethal, and many diagnoses are only established by postmortem. Thus only small series studies have been reported in patients with LV free wall rupture who were able to undergo surgery. Operative mortality can be as high as $30 \%$ in patients with contained rupture. Haddadin et al. showed the simple glued patch covering technique is superior than direct suture closure technique (hospital mortality $12 \%$ vs. $36 \%$ ) [7].

\subsection{Case Reports: Mechanical Complications of Acute Myocardial Infraction}

Wei Wang and Anson Cheung

\subsubsection{Clinical Case 1}

A 55-year-old male with no previous cardiac history presented late with an inferior STEMI. The patient developed acute heart failure and cardiogenic shock requiring intubation in emergency department. TTE showed a large posterior VSD involving the base of mitral valve and extending downward toward the apex. The patient had moderate to severe left ventricular dysfunction with an ejection fraction of $35 \%$.

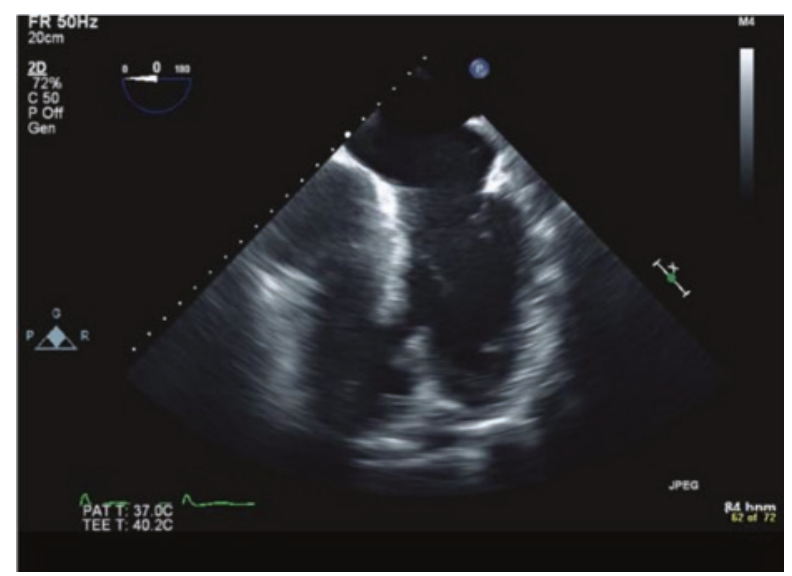




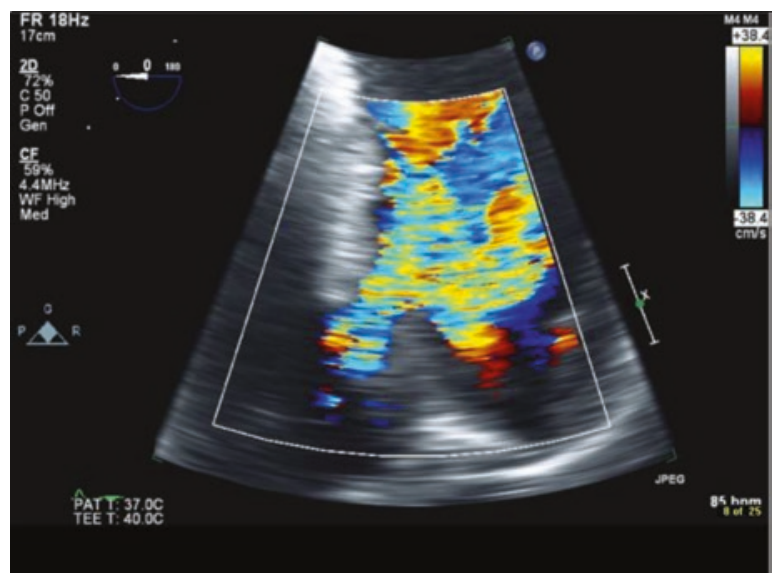

Coronary angiography showed an occluded RCA with no visible vessels distally, a small and diffusely diseased LAD, and LCx without any critical lesions. IABP was inserted in coronary suite. Despite escalating dosage of vasopressor and inotropes, the patient developed persistent cardiogenic shock with severe metabolic acidosis with a $\mathrm{pH}$ of 6.85 . Short-term MCS was initiated percutaneously in the form of VA ECMO via femoral vessels.

Metabolic derangement and hemodynamic was corrected with ECMO support in the next 3 days. Subsequently, the patient was taken to the operating theatre for VSD repair. Intraoperative findings include infarction of the entire inferior wall with involvement of the RV and an extensive VSD extending from the base of mitral annulus to the apex. A successful VSD repair using infarct exclusion technique with large bovine pericardial patches was carried out. There was no residual shunt detected post repair, and only mild MR was seen by transesophageal echocardiogram (TEE) postoperatively. Unfortunately, the patient was unable to be weaned from cardiopulmonary bypass due to moderate to severe RV dysfunction and pulmonary dysfunction. VA ECMO was reinitiated, and further support was required for another 8 days with eventual successful wean from ECMO.

\subsubsection{Clinic Case 2}

A 62-year-old female, who had missed acute STEMI 5 days previously, presented with cardiogenic shock and was brought to catheter laboratory. The coronary angiogram showed occluded LAD and left circumflex with LVEF 15\%. Balloon angioplasty was performed, but it was unable to restore LAD flow. The patient was hemodynamically unstable despite intubation, IABP, and high dose of norepinephrine and dobutamine. Echocardiogram showed severe MR and signs of tamponade secondary to large amount of pericardial fluid. Cardiac surgery was emergently consulted, and the patient was brought to the operating room for emergency ECMO. The patient had a cardiac arrest before cutting the skin. Stat cardiopulmonary bypass 
was established via median sternotomy. There was a lot of blood found in the pericardial cavity and LV free wall rupture was identified. The LV tissue around rupture area was necrotic, which made it impossible to sew on the fragile tissue. A large bovine pericardium patch was glued on the LV free wall. The patient was then placed on central ECMO and chest left open and was listed for high status heart transplant candidate. Fortunately, 3 days later a donor heart became available, and the patient received orthotopic heart transplant successfully. Posttransplant course was uneventful and she was discharged from hospital 3 weeks later.

\section{References}

1. Lawrence HC. Cardiac surgery in the adult. NY: McGraw-Hill Education; 2011.

2. Massetti M, et al. Postinfarction ventricular septal rupture: early repair through the right atrial approach. J Thorac Cardiovasc Surg. 2000;119(4 Pt 1):784-9.

3. Thiele $\mathrm{H}$, et al. Immediate primary transcatheter closure of postinfarction ventricular septal defects. Eur Heart J. 2009;30(1):81-8.

4. Thiele $\mathrm{H}$, et al. Intraaortic balloon support for myocardial infarction with cardiogenic shock. $\mathrm{N}$ Engl J Med. 2012;367(14):1287-96.

5. Arnaoutakis GJ, et al. Surgical repair of ventricular septal defect after myocardial infarction: outcomes from the Society of Thoracic Surgeons National Database. Ann Thorac Surg. 2012;94(2):436-43.

6. Lorusso R, Gelsomino S, De Cicco G, et al. Mitral valve surgery in emergency for severe acute regurgitation: analysis of postoperative results from a multicentre study. Eur J Cardiothorac Surg. 2008;33(4):573-82.

7. Haddadin S, et al. Surgical treatment of postinfarction left ventricular free wall rupture. J Card Surg. 2009;24(6):624-31.

Open Access This chapter is licensed under the terms of the Creative Commons Attribution 4.0 International License (http://creativecommons.org/licenses/by/4.0/), which permits use, sharing, adaptation, distribution and reproduction in any medium or format, as long as you give appropriate credit to the original author(s) and the source, provide a link to the Creative Commons license and indicate if changes were made.

The images or other third party material in this chapter are included in the chapter's Creative Commons license, unless indicated otherwise in a credit line to the material. If material is not included in the chapter's Creative Commons license and your intended use is not permitted by statutory regulation or exceeds the permitted use, you will need to obtain permission directly from the copyright holder.

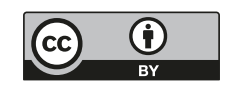

